\title{
Levantamento de indicadores de maturidade digital a partir de um mapeamento sistemático da literatura
}

\author{
Alex Rogério da Silva Calado ${ }^{1}$, Ricardo André Cavalcante de Souza ${ }^{1,2}$ \\ ${ }^{1}$ Programa de Pós-Graduação em Informática Aplicada (PPGIA) \\ ${ }^{2}$ Departamento de Computação (DC) \\ Universidade Federal Rural de Pernambuco (UFRPE) \\ alex.rogerio.calado@gmail.com, ricardo.souza@ufrpe.br
}

\begin{abstract}
Developing a digital transformation strategy is one of the main priorities of organizations that operate in the most diverse markets. Digital transformation is about delivering value and providing a better experience to customers through digital technologies. The digital transformation involves a profound change in organizational processes, business models and also in the behavior of the people involved, such as leaders, employees and even customers. The first step in drawing up a good strategy is to diagnose the organization's current situation, in this context, in terms of digital maturity. This work then presents a survey of the dimensions and indicators used in the diagnosis of the digital maturity level of organizations, resulting from a systematic literature mapping.
\end{abstract}

Resumo. Desenvolver uma estratégia de transformação digital é uma das principais prioridades de organizações que atuam nos mais diversificados mercados. A transformação digital consiste na entrega de valor e de uma melhor experiência aos clientes por meio de tecnologias digitais. A transformação digital envolve uma mudança profunda nos processos organizacionais, modelos de negócio e, também, no comportamento das pessoas envolvidas, como líderes, funcionários e até mesmo clientes. $O$ primeiro passo para se traçar uma boa estratégia é diagnosticar a situação atual da organização, neste contexto, em termos de maturidade digital. Este trabalho apresenta então um levantamento das dimensões e indicadores utilizados no diagnóstico do nível de maturidade digital das organizações, resultante de um mapeamento sistemático da literatura.

\section{Introdução}

Um levantamento recente [BDO 2019] com 300 executivos de empresas que atuam em segmentos de mercado diversificados, com faturamento anual entre 250 milhões a 3 bilhões de dólares, aponta que 34\% deles citam o desenvolvimento de uma estratégia de transformação digital como prioridade número um.

Segundo [Matt 2015], a Transformação Digital pode ser entendida como "um processo que visa melhorar uma entidade, provocando alterações significativas em suas propriedades por meio de combinações de tecnologias de informação, computação, comunicação e conectividade". A Transformação Digital representa os novos modelos de negócios, produtos e serviços, bem como novos tipos de experiências do cliente. A Transformação Digital não é um projeto, é um modo permanente de trabalhar, pensar e agir que busca explorar tecnologias digitais para criar melhor experiência. 
A Transformação Digital é essencialmente vinculada à entrega de experiência através de tecnologias digitais habilitadas pela Internet [Baxendale 2019]. A Transformação (ou adaptação) Digital pode também ser considerada um processo de aculturação pelo qual indivíduos, times e organizações são levados a mudar paulatinamente de comportamentos e estruturas analógicos para plataformas digitais [Meira e Neves 2019]. Plataformas representam a integração de métodos de trabalho, sistemas tecnológicos e modelos de negócios.

Os benefícios da Transformação Digital são múltiplos e incluem aumentos nas vendas ou na produtividade, inovações na criação de valor, além de novas formas de interação com os clientes. Como resultado, modelos de negócios inteiros podem ser remodelados ou substituídos [Downes and Nunes, 2013].

A Transformação Digital é uma jornada e incluí diferentes níveis de maturidade digital. Segundo Met et al. (2020), as organizações podem ser classificadas em quatro níveis de maturidade digital: (1) Iniciantes, não possuem pressa de mudar para outras formas de tecnologias digitais, como mídias sociais; (2) Conservadoras, com visão e estrutura eficazes em termos de adaptação à tecnologia; (3) Fashionistas, procuram adotar rapidamente tecnologias digitais, mas sem uma visão e estratégia claras que abranjam toda a empresa de maneira coordenada; e (4) Digirati, a liderança possui uma visão concreta, clara e bem entendida de como as tecnologias digitais alcançarão e se comprometerão efetivamente com essa visão.

Segundo um estudo da consultoria internacional de negócios Delloite (2018), um alto nível de maturidade digital está associado com melhor desempenho financeiro, pois permite às organizações: identificar e aproveitar novas oportunidades, desenvolver novas fontes de receita, responder com mais agilidade a demandas de clientes e mercado, e operar com maior eficiência.

Diante deste contexto, no qual as organizações precisam definir uma estratégia de Transformação Digital e que uma boa prática adotada em planejamento estratégico é inicialmente diagnosticar a situação atual da organização, este trabalho apresenta um levantamento das dimensões e indicadores usados para avaliar o nível de maturidade digital de organizações, resultante de um mapeamento sistemático da literatura.

Além desta seção introdutória, o artigo está organizado em mais 3 seções. A Seção 2 apresenta a sistemática do mapeamento da literatura. A Seção 3 descreve os resultados obtidos. Finalmente, na Seção 4 são realizadas as considerações finais.

\section{Mapeamento Sistemático da Literatura}

O mapeamento sistemático da literatura consiste de quatro etapas [Petersen et al. 2008]: (1) Definição do escopo do mapeamento, para definição das bases de pesquisa, período de publicação dos estudos primários e questão de pesquisa; (2) Busca por estudos primários, para definição da string de busca para direcionar a consulta nas bases de pesquisa; (3) Extração de dados, para filtro a partir da análise do título e resumo dos estudos primários retornados; e (4) Análise e síntese, para leitura completa e análise qualitativa dos estudos primários e síntese daqueles que contribuem com a resposta à questão de pesquisa formulada.. Nas próximas subseções é apresentada a execução de tais passos no contexto deste trabalho. 


\subsection{Definição do Escopo do Mapeamento}

Esta etapa consistiu no planejamento do mapeamento da literatura, o qual resultou em um Protocolo de Pesquisa (Tabela 1) sintetizando a estratégia de busca e uma Questão de Pesquisa (RQ do inglês Research Question) para direcionar a análise dos estudos.

Tabela1. Protocolo do Mapeamento Sistemático

Questão de Pesquisa: Quais os fatores de mensuração ou avaliação da maturidade digita de uma organização?

\begin{tabular}{|c|c|}
\hline \multicolumn{2}{|c|}{ Estratégia de Busca } \\
\hline Bases de dados científicas & Equipe Executora \\
\hline $\begin{array}{l}\text { IEEE Xplore Digital Library; Springer Link; } \\
\text { ACM Digital Library; Scielo. }\end{array}$ & $\begin{array}{l}\text { 1. Autor (estudante de pós-graduação); } \\
\text { 2. Professor orientador. }\end{array}$ \\
\hline Palavras-chave & Keywords \\
\hline $\begin{array}{l}\text { 1. "MATURIDADE DIGITAL" } \\
\text { 2. "TRANSFORMAÇÃO DIGITAL" } \\
\text { 3. "AVALIAÇÃO" } \\
\text { 4. "MENSURA" } \\
\text { 5. "INDICADOR" }\end{array}$ & $\begin{array}{l}\text { 6. "DIGITALLY MATURING" } \\
\text { 7. "DIGITAL TRANSFORMATION" } \\
\text { 8. "EVALUATE" } \\
\text { 9. "MEASURE" } \\
\text { 10. "INDICATOR" }\end{array}$ \\
\hline
\end{tabular}

Método de Execução da Busca

1. Pesquisar nas bases de dados científicas por meio da string de pesquisa que consiste na combinação das palavras-chave através de operadores lógicos;

2. Refinar a busca com filtro pelo ano de publicação entre 2015 e 2020;

3. Analisar os títulos e resumos dos estudos primários (EP) retornados nas buscas e selecionar aqueles que atendem os critérios de inclusão e exclusão;

4. Analisar o texto dos EP selecionados e aplicar os critérios de qualidade para identificar os aceitos e rejeitados para o contexto do mapeamento sistemático da literatura.

\subsection{Busca por Estudos Primários}

Nesta etapa foram realizadas buscas nas bases de dados científicas com o intuito de obter estudos publicados. Os estudos resultantes de uma busca visando responder hipóteses ou questões de pesquisa caracterizam-se como Estudos Primários (EP) [Magalhães et al. 2013]. A construção da string de busca (Tabela 2) consistiu na utilização das palavras-chave com os conectores lógicos OR e AND.

Tabela 2: String de Busca

\begin{tabular}{|c|c|}
\hline ("Evaluate”OR “Measure" & ("Avaliação"OR “Mensuração" \\
OR "Indicator") AND ("Digital & OR “Indicador") AND (“Maturidade \\
Maturity" OR Digital Transformation) & Digital" OR "Transformação Digital") \\
\hline
\end{tabular}

Como resultado, foram obtidos $124 \mathrm{EP}$, classificados e organizados através da ferramenta de software Mendeley (mendeley.com), software que pode ser utilizado como gerenciador e repositório de trabalhos científicos, havendo a possibilidade de compartilhar, criar comentários e editar dados dos artigos [Zaugg et al. 2011]. Também, existe a possibilidade de integração com motores de busca, facilitando a pesquisa, melhorando a organização e a avaliação dos artigos encontrados. A Tabela 3 apresenta os EPs resultantes na busca por ano de publicação e pela fonte. 
Tabela 3. Quantitativo de EP retornados na etapa inicial de busca

\begin{tabular}{|l|c|c|c|c|c|c|}
\hline \multirow{2}{*}{ Base de Busca } & \multirow{2}{*}{ Quantidade de EPs } & \multicolumn{5}{c|}{ Ano de Publicação } \\
\cline { 3 - 8 } & & 2016 & 2017 & 2018 & 2019 & 2020 \\
\hline IEEE & 32 & - & 6 & 12 & 14 & - \\
\hline Springer & 71 & 2 & 10 & 17 & 36 & 6 \\
\hline ACM & 21 & 1 & 5 & 6 & 9 & - \\
\hline Scielo & 2 & 2 & - & - & - & - \\
\hline Quantidade Total de EP & $\mathbf{1 2 4}$ & $\mathbf{5}$ & $\mathbf{2 1}$ & $\mathbf{3 5}$ & $\mathbf{5 9}$ & $\mathbf{6}$ \\
\hline
\end{tabular}

\subsection{Extração de Dados}

Nesta etapa foi realizada a análise do título e resumo dos EP e aplicados os seguintes critérios exclusão: (1) EP escrito em outra língua que não a inglesa e portuguesa; (2) EP não relacionado ao tema da pesquisa; (3) EP repetido, usando a ferramenta Mendeley os resultados foram ordenados por ano de publicação e por ordem alfabética e descartadas as duplicações; e (4) EP enquadrado como resumo, curso, tutorial, workshop e afins.

A Tabela 4 apresenta o quantitativo de EP selecionados na extração de dados de modo a permitir comparação com a etapa anterior (Tabela 3). Vale ressaltar que houve significativa redução de EP selecionados nesta etapa, devido principalmente ao critério de exclusão de não alinhamento com o tema da pesquisa.

Tabela 4. Quantidade de EP selecionados na Extração de Dados

\begin{tabular}{|l|c|c|c|c|c|c|}
\hline & & \multicolumn{5}{|c|}{ Ano de Publicação } \\
\cline { 3 - 8 } Base de Busca & Quantidade de EP & $\mathbf{2 0 1 6}$ & $\mathbf{2 0 1 7}$ & $\mathbf{2 0 1 8}$ & $\mathbf{2 0 1 9}$ & $\mathbf{2 0 2 0}$ \\
\hline IEEE & 8 & - & 3 & 4 & 1 & - \\
\hline Springer & 23 & 1 & 2 & 4 & 13 & 3 \\
\hline ACM & 5 & - & - & 2 & 3 & - \\
\hline Quantidade Total de EP & $\mathbf{3 6}$ & $\mathbf{1}$ & $\mathbf{5}$ & $\mathbf{1 0}$ & $\mathbf{1 7}$ & $\mathbf{3}$ \\
\hline
\end{tabular}

\subsection{Análise e Síntese}

Os EPs selecionados ${ }^{1}$ foram analisados qualitativamente através dos seguintes critérios qualitativos (CQ): (1) EP identifica fatores que colaborem para uma transformação digital de sucesso; (2) EP aponta indicadores que contribuem para classificação do nível de transformação digital; (3) EP trata de pontos chave quanto a metodologia de avaliação de transformação digital/maturidade digital; (4) EP põe em prática ou construiu pelo menos um modelo ou framework de classificação do nível de transformação digital ou maturidade digital de uma organização; (5) EP aborda pontos e/ou traz conceitos relevantes sobre transformação digital ou maturidade digital.

Após a leitura completa de cada um dos 36 EPs selecionados na etapa anterior, foram atribuídos AT (Atende) ou NA (Não Atende) para os CQ. Foi então possível calcular o Índice de Aceitação (IA) do EP de acordo com a quantidade de CQ atendidos, ou seja, o IA é a somatória de CQ com valor AT. Os EP com IA igual ou maior a 3 foram considerados Artigos Aceitos (AA), os demais Artigos Rejeitados (AR) para o propósito deste mapeamento. A Tabela 5 apresenta a identificação e análise qualitativa dos EP avaliados, destacando através de sombreamento os EP considerados aceitos.

\footnotetext{
${ }^{1}$ As referências completas podem ser consultadas em https://bit.ly/39te10Q
} 
Tabela 5. Síntese da Análise Qualitativa dos Estudos Primários (EP) ${ }^{2}$

\begin{tabular}{|c|c|c|c|c|c|c|c|c|}
\hline ID & Título & CQ1 & CQ2 & CQ3 & CQ4 & CQ5 & IA & Resultado \\
\hline 01 & Römer et al. 2017 & AT & NA & NA & NA & AT & 2 & AR \\
\hline 02 & Venkateswaran, 2017 & AT & AT & NA & NA & AT & 3 & AA \\
\hline 03 & Wilker et al. 2018 & AT & NA & NA & NA & NA & 1 & $\mathrm{AR}$ \\
\hline 04 & Ebrahimi 2018 & AT & NA & NA & NA & AT & 2 & AR \\
\hline 05 & Al-Ruithe 2017 & AT & NA & AT & NA & NA & 3 & AR \\
\hline 06 & Seitz, 2018 & AT & AT & AT & AT & AT & 5 & AA \\
\hline 07 & Haoud, 2019 & NA & NA & NA & NA & NA & 0 & AR \\
\hline 08 & Grivas et al. 2018a & AT & NA & NA & NA & AT & 2 & AR \\
\hline 09 & Saini 2018 & NA & NA & NA & NA & AT & 1 & AR \\
\hline 10 & Löffler et al. 2018 & NA & NA & AT & NA & AT & 2 & AR \\
\hline 11 & Haddud 2018 & AT & AT & AT & NA & AT & 4 & AA \\
\hline 12 & Lederer, 2018 & AT & NA & AT & NA & AT & 3 & AA \\
\hline 13 & Prakash 2019 & AT & NA & NA & NA & AT & 2 & AR \\
\hline 14 & Pradeepkumar et al. 2019 & NA & NA & NA & NA & NA & 0 & AR \\
\hline 15 & Bley 2019 & NA & AT & AT & AT & AT & 4 & AA \\
\hline 16 & Graf 2018 & AT & AT & AT & AT & AT & 5 & AA \\
\hline 17 & Carolis et al. 2017 & AT & AT & AT & AT & AT & 5 & $\mathrm{AA}$ \\
\hline 18 & Elsäßer 2019 & AT & AT & AT & AT & AT & 5 & AA \\
\hline 19 & Grivas 2018b & AT & NA & AT & AT & NA & 3 & AA \\
\hline 20 & Goerzig et al. 2018 & AT & NA & NA & NA & AT & 2 & $\mathrm{AR}$ \\
\hline 21 & Voß, 2019 & AT & NA & AT & NA & AT & 3 & AA \\
\hline 22 & Van Looy 2017 & AT & NA & NA & NA & NA & 1 & AR \\
\hline 23 & Bonanomi 2019 & AT & NA & NA & NA & AT & 2 & AR \\
\hline 24 & Brozzi et al. 2018 & AT & AT & AT & AT & AT & 4 & AA \\
\hline 25 & Delone, 2020 & AT & NA & NA & NA & AT & 2 & AR \\
\hline 26 & Met et al. 2020 & AT & AT & AT & NA & NA & 3 & AA \\
\hline 27 & Bonanomi 2019 & AT & AR & AR & AR & AT & 2 & AR \\
\hline 28 & Greff et al. 2018 & $\mathrm{AR}$ & $\mathrm{AR}$ & AR & AR & AT & 1 & $\mathrm{AR}$ \\
\hline 29 & Guarino et al. 2019 & NA & NA & AT & AR & AT & 2 & AR \\
\hline 30 & Murawski et al. 2019 & NA & NA & NA & NA & AT & 1 & $\mathrm{AR}$ \\
\hline 31 & Kokkinakos et al. 2016 & NA & AT & AT & NA & AT & 3 & AA \\
\hline 32 & Tadeu et al. 2019 & AT & AT & AT & NA & AT & 4 & AA \\
\hline 33 & Şen 2018 & AT & NA & NA & NA & AT & 2 & AR \\
\hline 34 & Arkhipova 2018 & AT & NA & $\mathrm{NA}$ & NA & AT & 2 & AR \\
\hline 35 & Ifenthaler 2020 & AT & AT & AT & NA & AT & 4 & AA \\
\hline 36 & Brown 2019 & AT & AT & AT & NA & AT & 4 & AA \\
\hline
\end{tabular}

\section{Resultados}

Após a análise qualitativa e posterior síntese (Tabela 6), pode-se observar que $16 \mathrm{EP}$ foram considerados aceitos, sendo então esses a serem utilizados para formulação da resposta à RQ elaborada. A Tabela 6 apresenta uma comparação quantitativa dos EP analisados e considerados aceitos e rejeitados entre as etapas do mapeamento.

Tabela 6. Comparação quantitativa entre as etapas do mapeamento

\begin{tabular}{|l|c|c|c|}
\hline \multicolumn{1}{|c|}{ Etapa } & EP analisados & EP rejeitados & EP aceitos \\
\hline Extração de Dados & 126 & $90(71.43 \%)$ & $36(28.57 \%)$ \\
\hline Análise Qualitativa & 36 & $20(55.55 \%)$ & $16(44.45 \%)$ \\
\hline
\end{tabular}

De modo a subsidiar a resposta à RQ, na Tabela 7 é apresentada a síntese das contribuições dos EPs considerados aceitos na etapa de análise qualitativa. 
Tabela 7. Síntese das Contribuições do EP

\begin{tabular}{|c|c|}
\hline ID & Descrição do estudo \\
\hline 02 & $\begin{array}{l}\text { Foi argumentado que o desempenho de organizações diferem entre públicas e privadas } \\
\text { devido aos seus ambientes institucionais distintos, o que leva a diferenças nos } \\
\text { resultados de desempenho. Foram propostas quatro proposições como critérios de } \\
\text { avaliação de desempenho: (a) responsabilidade das partes interessadas, (b) agilidade, } \\
\text { (c) inovação e, (d) compromisso com a conclusão. }\end{array}$ \\
\hline 06 & $\begin{array}{l}\text { Identifica valor digital e grau de maturidade digital a partir de entrevistas com } \\
\text { especialistas em transformação digital. Foram identificadas } 22 \text { dimensões, não } \\
\text { discriminadas, agrupadas em quatro conjuntos: Modelos de negócios digitais, } \\
\text { Operações de negócios digitais, Marketing Digital e Estratégia de Vendas, Mentalidade } \\
\text { digital. Um dos resultados é que Mentalidade Digital tem um bom potencial de criação } \\
\text { de valor digital, mas é o grupo com menor grau de maturidade. Demonstra que } \\
\text { iniciativas, que visam estabelecer uma cultura entre os funcionários, aberta a ideias } \\
\text { digitais e sem reservas sobre tecnologias modernas, devem ser intensificadas. }\end{array}$ \\
\hline 11 & $\begin{array}{l}\text { Apresenta os principais elementos de um local de trabalho digital (rede de colaboração, } \\
\text { tecnologia, controle e drivers de negócio) e possíveis benefícios e desafios relacionados } \\
\text { aos locais de trabalho digitais. Indicou que os líderes devem estar presentes em todos } \\
\text { os espaços digitais, entender quem eles estão liderando e liderar pela persuasão, } \\
\text { adaptar o gerenciamento de fluxo e, serem "humanizados" como habilidades } \\
\text { importantes nos locais de trabalho digitais. Aponta ainda vários pontos-chave de } \\
\text { sucesso para o gerenciamento eficaz dos locais de trabalho digitais. }\end{array}$ \\
\hline 12 & $\begin{array}{l}\text { Foi mostrado que o S-BPM (Subject-oriented Business Process Management) é } \\
\text { adequado quanto à digitalização do gerenciamento de processos de negócios. Para a } \\
\text { avaliação foi feita uma adaptação de uma estrutura, com base em três domínios: (i) } \\
\text { desenvolvimento de produtos digitais, (ii) produção digital e (iii) transformação digital } \\
\text { dos negócios. O trabalho corrobora com indicação de determinantes de avaliação digital } \\
\text { com embasamento teórico. }\end{array}$ \\
\hline 15 & $\begin{array}{l}\text { A partir das evidências apresentadas na literatura é proposto um modelo de maturidade } \\
\text { que usa o conceito de funções para representar componentes específicos das } \\
\text { empresas para obter um modelo de maturidade mais flexível e escalável para pequenas } \\
\text { e médias empresas (PME). A maturidade digital é demonstrada gráfica e } \\
\text { aritmeticamente e introduz uma abordagem de avaliação para definir se o aumento da } \\
\text { digitalização é realmente relevante e vale a pena para a empresa. }\end{array}$ \\
\hline 16 & $\begin{array}{l}\text { O estudo indica o framework estruturado Transformation Compass como método de } \\
\text { apoio e liderança ideal na transformação digital para PME. Identifica os principais } \\
\text { frameworks do mercado (Digital innovation and transformation framework, Digital } \\
\text { Transformation Framework, Digital Portfolio, McKinsey Model, Digital Transformation } \\
\text { Framework e Digital Maturity Model) e se baseia no CMMI (Capability Maturity Model } \\
\text { Integration) para classificação de níveis de maturidade digital de PME. }\end{array}$ \\
\hline 17 & $\begin{array}{l}\text { Propõe um modelo para estabelecer as bases para a investigação da maturidade digital } \\
\text { de empresas de manufatura. Diferentes dimensões (Processo, Monitoramento e } \\
\text { Controle, Tecnologia e Organização) são usadas para avaliar cinco dos principais } \\
\text { processos de fabricação: (1) projeto e engenharia, (2) gerenciamento de produção, (3) } \\
\text { gerenciamento de qualidade, (4) gerenciamento de manutenção e (5) gerenciamento de } \\
\text { logística. Um método de pontuação para avaliação da maturidade é subsequentemente } \\
\text { definido, baseado na classificação de níveis usados pelo CMMI, a fim de identificar os } \\
\text { pontos críticos na implementação da transformação digital. }\end{array}$ \\
\hline 18 & $\begin{array}{l}\text { Propõe um modelo conceitual que estrutura o a maturidade digital em quatro fases: (1) } \\
\text { Inicial: conversão de analógico para código binário, armazenamento eletrônico de } \\
\text { dados; (2) Convergência de Mídia: fusão de vários formatos de dados usando } \\
\text { plataforma ou dispositivo; (3) Digitalização: uso de inovação técnica resultante das fases } \\
\text { anteriores; (4) Transformação Digital: criação de um ecossistema generativo de itens } \\
\text { técnicos e sociais resultando em mudanças no Modelo de Negócios. }\end{array}$ \\
\hline 19 & $\begin{array}{l}\text { Foram realizadas avaliações em empresas alemãs nas áreas: Estratégia, Organização, } \\
\text { Pessoas e Tecnologia. O agrupamento de semelhanças de empresas inovadoras levou } \\
\text { a quatro papéis principais que promovem o desenvolvimento ágil bem-sucedido de }\end{array}$ \\
\hline
\end{tabular}




\begin{tabular}{|c|c|}
\hline & $\begin{array}{l}\text { produtos digitais: Liderança Digital, Nativos Digitais, Aceleradores Digitais e Clientes } \\
\text { Digitalizados. Foi descrito como cada função aprimora a agilidade na empresa e garante } \\
\text { o sucesso no decorrer do processo de transformação digital. }\end{array}$ \\
\hline 21 & $\begin{array}{l}\text { Detalha alguns dos principais frameworks de avaliação de maturidade digital, tais como, } \\
\text { Digital Maturity Model, } 360^{\circ} \text { OmniSales model e The transformation model. Foram } \\
\text { identificadas lacunas quanto à classificação da transformação digital e foram sugeridas } \\
\text { pesquisas sobre a operacionalização detalhada dos diferentes fatores que influenciam o } \\
\text { processo de mudança e observações da maturidade digital em longo prazo. É apontada } \\
\text { a oportunidade de desenvolver um framework amigável que compreenda os aspectos } \\
\text { cognitivos e afetivos que são necessários para o desenvolvimento organizacional. } \\
\text { Assim um enfoque maior nos funcionários resultaria em uma avaliação mais abrangente } \\
\text { da maturidade digital de uma organização. }\end{array}$ \\
\hline 24 & $\begin{array}{l}\text { Depois de coletar e definir categorias em termos de características das ferramentas } \\
\text { individuais de autoavaliação e considerando desafios específicos das PMEs em projetos } \\
\text { de transformação digital, foi projetada a estrutura de uma ferramenta de autoavaliação } \\
\text { com três dimensões (Produção e Operações, Digitalização e, Ecosistema) e } 23 \text { itens. A } \\
\text { estrutura teve como base alguns frameworks, como Reifegradmodell Maturity Model, } \\
\text { The Digital Maturity Check, Digitalisation Index e, Benchmarking Readiness. }\end{array}$ \\
\hline 31 & $\begin{array}{l}\text { Com enfoque no setor Público, usa o Índice de Adoção Digital (DAI) e o Índice de } \\
\text { Evolução Digital (DEI) para quantificar o nível de progresso na transformação digital em } \\
\text { cinco governos diferentes. Os resultados mostraram que o setor público não apenas } \\
\text { acompanha o setor privado em termos de digitalização, mas em três (dos cinco) casos } \\
\text { lidera a corrida. São ainda identificados três níveis de maturidade (Iniciante, Em } \\
\text { desenvolvimento e, Maduro) em relação à transformação digital dos governos e cinco } \\
\text { dimensões que moldam o caminho para a transformação digital: estratégia, liderança, } \\
\text { habilidades da força de trabalho, cultura digital e foco no usuário. }\end{array}$ \\
\hline 32 & $\begin{array}{l}\text { Analisa a transformação digital no cenário de empresas brasileiras. Foi proposto um } \\
\text { modelo baseado em dez dimensões para definir maturidade digital: Cultura, Novos } \\
\text { Modelos de Negócio, Riscos e Investimentos, Ética e Aspectos Legais, Estratégia } \\
\text { Digital, Tecnologias Digitais, Analítica, Aspectos Estratégicos e Processo Digital, Cadeia } \\
\text { de Valor, Necessidades do Cliente. Este estudo revelou que, para a maioria das } \\
\text { empresas entrevistadas, a digitalização é considerada apenas a mudança de processos } \\
\text { analógicos, manuais ou interventivos, para versões baseadas em tecnologias digitais. }\end{array}$ \\
\hline 33 & $\begin{array}{l}\text { Aponta os principais fatores que devem ser gerenciados com eficiência e que trazem } \\
\text { sucesso durante a transformação digital: gestão estratégica, marketing, cultura } \\
\text { corporativa e recursos humanos. Mostra ainda que a era da digitalização, deve ter cinco } \\
\text { fases contínuas nas empresas: ambição, design, entrega, escala e refinamento. }\end{array}$ \\
\hline 35 & $\begin{array}{l}\text { A partir de uma sinopse de seis modelos de maturidade, foi desenvolvido um modelo de } \\
\text { maturidade da adoção de tecnologia para organizações educacionais (MMEO). O } \\
\text { modelo possui seis dimensões (infraestrutura, estratégia e liderança, organização, } \\
\text { funcionários, cultura e tecnologia educacional) e cinco níveis de maturidade digital } \\
\text { (minimalista, conservador, pragmático, avançado, e pioneiro). }\end{array}$ \\
\hline 36 & $\begin{array}{l}\text { Descreve como as organizações podem efetivamente alcançar a transformação digital } \\
\text { com base em uma estratégia comercial digital definida. Por meio de uma revisão da } \\
\text { literatura, demonstrou os conceitos fundamentais que vinculam a estratégia de negócios } \\
\text { digitais à transformação digital (TD): tecnologias digitais, estratégia e modelo de } \\
\text { negócios digitais, estratégia de TD, liderança, transparência e maturidade digital. }\end{array}$ \\
\hline
\end{tabular}

Tendo em vista que traçar uma estratégia de Transformação Digital (TD) é uma das prioridades de organizações da economia baseada em conhecimento [BDO 2019], a Figura 1 apresenta um modelo conceitual que sintetiza as dimensões organizacionais que influenciam e devem ser consideradas na estratégia de TD, visando o alcance de níveis incrementais de maturidade digital. Há uma correlação direta entre os componentes do modelo, quanto mais valor digital é entregue nas dimensões, maior é a maturidade digital da organização (Seitz and Burosch, 2018). Valor digital refere-se a usar tecnologias digitais como meio para melhorar a experiência dos clientes ou como 
eles são servidos (Mckinsey, 2015). O modelo conceitual foi elaborado a partir das descobertas do mapeamento realizado. Os estudos utilizados como fonte para definição do modelo são referenciados pelo identificador, conforme descrito na Tabela 5.

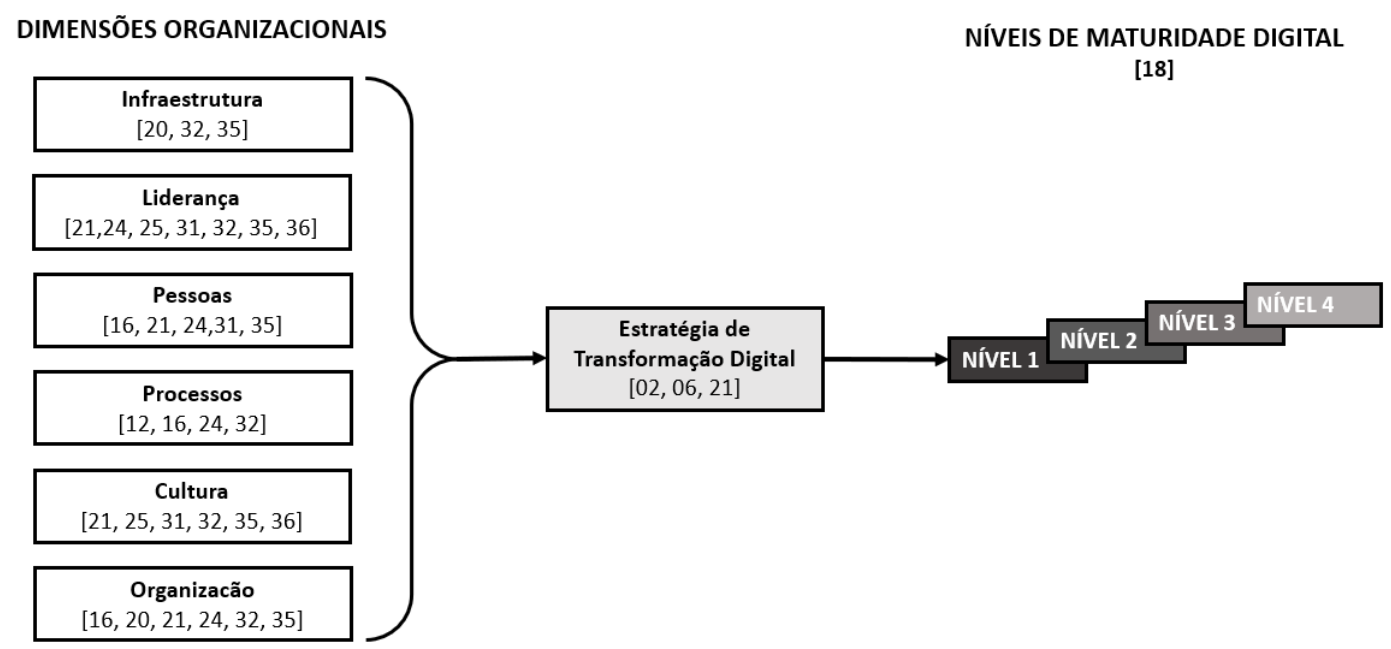

Figura 1. Modelo conceitual para uma estratégia de Transformação Digital

As dimensões organizacionais usadas no modelo conceitual foram as mais recorrentes encontradas no recorte do mapeamento da literatura: Infraestrutura: engloba os recursos e tecnologias digitais em geral, pode ser representada como a camada base de suporte tecnológico para a transformação digital; Liderança: corresponde a estratégia e ao modelo de gerenciamento utilizado pelos líderes e como ele se posiciona dentro da organização; Processos: não apenas do ponto de vista operacional, mas também da criação e gerenciamento corporativo do uso e importância de dados produzidos dentro da organização; Pessoas: diz respeito à qualificação, mentalidade e competência digital dos funcionários para aprender e aplicar novas tecnologias e conhecimentos digitais; Cultura: aborda como os funcionários interagem e se comunicam entre si e como as informações e conhecimentos são compartilhados; Organizacional: trata da gestão de investimentos na inovação, de práticas e recursos da empresa.

De modo a responder à RQ, conforme descrito no protocolo (Tabela 1), os principais indicadores associados a cada dimensão organizacional, identificados a partir do mapeamento da literatura, estão consolidados na Tabela 8, com as respectivas fontes referenciadas pelo identificador. No contexto deste trabalho, os indicadores mensuram e evidenciam a maturidade digital de uma organização.

Tabela 8. Indicadores de maturidade digital por dimensão organizacional

\begin{tabular}{|c|l|}
\hline $\begin{array}{c}\text { Dimensão } \\
\text { Organizacional }\end{array}$ & \multicolumn{1}{|c|}{ Indicadores de maturidade digital } \\
\hline Infraestrutura & $\begin{array}{l}\text { Infraestrutura atualizada de dispositivos digitais e softwares; cenário de } \\
\text { tecnologia homogêneo [35]; uso de novas e diversificadas tecnologias } \\
\text { digitais integradas (Segurança Digital, Internet das Coisas, Computação em } \\
\text { Nuvem, IA, etc.) [25,20, 21] }\end{array}$ \\
\hline Liderança & $\begin{array}{l}\text { Existência e implementação de uma estratégia digital; os dirigentes } \\
\text { promovem a digitalização com prioridade; análise de novas tecnologias; } \\
\text { estilo de liderança democrática; liberdade criativa concedida [36]; visão } \\
\text { focada na transformação fundamental do processo [31]; controle } \\
\text { centralizado; uso de facilitadores orientados a análise e serviços [18]; } \\
\text { liderança ciente e sofisticada digitalmente [31] }\end{array}$ \\
\hline
\end{tabular}




\begin{tabular}{|c|l|}
\hline Pessoas & $\begin{array}{l}\text { Funcionários com conhecimentos/habilidades em lidar com tecnologias } \\
\text { digitais; uso de dispositivos e serviços; prontidão para treinamento adicional } \\
\text { [36]; investimento em capacitação e qualificação dos funcionários [24, 31]; } \\
\text { preenchimento da lacuna de talentos [25] }\end{array}$ \\
\hline Cultura & $\begin{array}{l}\text { Abertura a novas tecnologias; abertura para mudança; comunicação } \\
\text { aberta; apoio mútuo [36]; tolerância a falhas [25,31]; organização ágil e } \\
\text { adaptável [25]; promoção da inovação e colaboração [18, 31] }\end{array}$ \\
\hline Processos & $\begin{array}{l}\text { Previsibilidade; adaptabilidade; flexibilidade; automação; controle; robustez; } \\
\text { tempo do ciclo do processo; grau de complexidade [12] }\end{array}$ \\
\hline Organizacional & $\begin{array}{l}\text { Recursos financeiros suficientes; suporte técnico (prestadores de serviços } \\
\text { internos e externos); compras e manutenção eficientes [36] }\end{array}$ \\
\hline
\end{tabular}

\section{Conclusão}

Este trabalho explorou conceitos importantes para a estratégia de transformação digital. Através de revisão sistemática da literatura foi possível fazer uma síntese das dimensões organizacionais que influenciam e devem ser consideradas na estratégia de transformação digital, bem como alguns dos principais indicadores associados. As dimensões organizacionais têm perspectivas técnica e humana. A perspectiva técnica refere-se à Infraestrutura, Processos e Organizacional. Já a perspectiva humana, referese à Liderança, Cultura e Pessoas.

Uma boa estratégia de transformação digital leva à organização a alcançar maiores níveis de maturidade digital. Quanto maior o nível de maturidade digital, maior é o desempenho financeiro da organização [Delloite 2018]. Os indicadores levantados, associados às dimensões organizacionais, servem para nortear, mensurar e evidenciar o nível de maturidade digital de uma organização.

Os resultados da pesquisa apontam que existem espaços pouco explorados no contexto da formulação da estratégia e do processo de transformação digital, bem como da avaliação da maturidade digital. Notou-se que há uma variedade razoável de frameworks capazes de auxiliar na avaliação de organizações quanto ao nível de maturidade digital, e que boa parte deles podem ser adaptados a contextos específicos, porém nenhum deles enfatiza o aspecto cognitivo e humana da transformação digital.

A partir do mapeamento da literatura, foi possível identificar que a perspectiva humana na transformação digital, em geral, recebe menos atenção do que a perspectiva técnica, apesar de ser determinante para o alcance de um maior nível de maturidade digital pelas organizações. Desta forma, entre as possibilidades de trabalho futuro estão: investigar as habilidades (skills) necessárias aos líderes, equipes e membros de equipes para atuar nos processos de transformação digital das organizações do conhecimento; aprofundar o estudo sobre cada uma das dimensões organizacionais identificadas, visando identificar lacunas de pesquisas; e realizar um estudo apenas da realidade local e nacional sobre o nível de maturidade digital das organizações.

\section{Referências}

Baxendale, G. (2019) Digital Transformation isn't that Technical, ITNOW, v.61, Issue 2, p. 04-05 https://doi.org.ez19.periodicos.capes.gov.br/10.1093/itnow/bwz030 
BDO. (2019) Building tomorrow's business: pioneering digital transformation in 2019. Disponível em <https://www.bdo.com/insights/business-financial-advisory/strategy,technology-transformation/digital-transformation-survey/business,-reinventedpioneering-digital-transform >. Acesso em 19 de março de 2020.

Delloite. (2018) Pivoting to digital maturity: seven capabilities central to digital transformation. Disponível em: $<$ https://www2.deloitte.com/content/dam/insights/us/articles/4955_Pivoting-todigital-maturity/DI_Pivoting-to-digital-maturity.pdf $>$. Acesso em 19 de março de 2020 .

Hustad, E. et al. (2019) Creating Business Value from Cloud-Based ERP Systems in Small and Medium-Sized Enterprises. In: Conference on e-Business, e-Services and e-Society. Springer, Cham. p. 691-703.

J. Seitz and A. Burosch, "Digital Value Creation" 2018 IEEE International Conference on Engineering, Technology and Innovation (ICE/ITMC), Stuttgart, 2018, pp. 1-5.

Magalhães, C., Santos, R., Silva, F. and Gomes, A. (2013). Caracterizando a pesquisa em informática na educação no Brasil: um mapeamento sistemático das publicações do SBIE. In Brazilian Symposium on Computers in Education (Simpósio Brasileiro de Informática na Educação-SBIE) (Vol. 24, No. 1, p. 22).

Matt, C.; Hess, T.; Benlian, A. (2015) Digital transformation strategies. Business \& Information Systems Engineering, v. 57, n. 5, p. 339-343.

Mckinsey. (2015) What 'digital' really means. Disponível em: $<$ https://www.mckinsey.com/industries/technology-media-and-telecommunications/ our-insights/what-digital-really-means\#>

Meira, S.; Neves, A. (2019) Strateegia - framework de transformação digital. The Digital Strategy Company. Disponível em: <https://www.tds.com.pe/framework/> Acesso em 20 de março de 2020.

Met, İ. et al. (2020) Key Success Factors for Strategic Management in Digital Business. In: Digital Business Strategies in Blockchain Ecosystems. Springer. p. 283-304.

Nambisan, S. et al. (2019) The digital transformation of innovation and entrepreneurship: Progress, challenges and key themes. Research Policy, v. 48, n. 8, p. 103773.

Petersen, K.; Feldt, R.; Mujtaba, S. and Mattsson, M. (2008) Systematic Mapping Studies in Software Engineering. 12th International Conference on Evaluation and Assessment in Software Engineering (EASE). University of Bari, Italy.

Venkateswaran, V.; Jyotishi, A. (2017) Digital Strategy Performance Differential Between Government and Private Sector: An New Institutional Economics Perspective. In: 2017 IEEE International Conference on Computational Intelligence and Computing Research (ICCIC). IEEE. p. 1-5.

Vianna, Maurício. Design thinking: inovação em negócios. Design Thinking, 2012.

Zaugg, H., West, R., Tateishi, I. and Randall, D. (2011). Mendeley: Creating communities of scholarly inquiry through research collaboration. Tech Trends, 55(1), 32-36; 